\title{
Uso de lactonas macrocíclicas para el control de la garrapata Rhipicephalus (Boophilus) microplus en el ganado bovino
}

\author{
Use of macrocyclic lactones to control the cattle tick Rhipicephalus (Boophilus) microplus \\ RI Rodríguez-Vivas*, RJ Arieta-Román, LC Pérez-Cogollo, \\ JA Rosado-Aguilar, GT Ramírez-Cruz, G Basto-Estrella
}

Facultad de Medicina Veterinaria y Zootecnia, Universidad Autónoma de Yucatán, Yucatán, México.

\begin{abstract}
SUMMARY
An overview of the macrocyclic lactones (ML), with recent results and their effect on the control of Rhipicephalus (Boophilus) microplus, is presented in this paper. This review describes in detail the chemistry, pharmacokinetics and dose presentation, security and time withdrawal and environmental safety of the groups belonging to ML: avermectins (ivermectin, doramectin, eprinomectin, abamectin) and milbemycin (moxidectin). There is also a discussion on their efficacy and the problem of resistance of $R$. (B.) microplus to ML. Finally, it is concluded that due to their high lipid solubility ML are widely distributed in tissues, being effective in the control of $R$. (B.) microplus in cattle. The efficacy of short-acting ivermectin, doramectin, eprinomectin, abamectin and moxidectin are similar (>90\% effective after four weeks post treatment, PT) to control adult stages of $R$. (B.) microplus, however, in long-acting LM are available the market and present efficiency of $>95 \%$ with persistence up to 70 days PT.

Palabras clave: Rhipicephalus (Boophilus) microplus, lactonas macrocíclicas, control, bovinos.

Key words: Rhipicephalus (Boophilus) microplus, macrocyclic lactones, control, cattle.
\end{abstract}

\section{INTRODUCCIÓN}

Uno de los principales problemas económicos en la ganadería bovina de las regiones tropicales y subtropicales de Centro y Sudamérica, África y Australia son las garrapatas y las enfermedades que éstas transmiten. Por su importancia económica y sanitaria la garrapata Rhipicephalus (Boophilus) microplus es uno de los principales ectoparásitos que causan graves problemas en la ganadería de pastoreo. Su efecto directo está relacionado con el daño a las pieles por acción de las picaduras, pérdida de sangre y el efecto de sus toxinas, lo cual incide negativamente sobre la ganancia de peso y producción de leche de animales infestados, mientras que su efecto indirecto está dado por la transmisión de agentes patógenos: Babesia bovis, Babesia bigemina y Anaplasma marginale, los cuales a su vez producen baja en la condición física, mortalidades y altos costos para su control (RodríguezVivas y col 2005).

Los métodos de control de garrapatas se clasifican en químicos (ixodicidas o garrapaticidas) y no químicos (vacunas y control biológico). El método más utilizado para el control de $R$. (B.) microplus es el uso de productos

Aceptado: 18.11.2009.

* km 15.5 carretera Mérida - Xmatkuil, CP 97 100, Mérida, Yucatán, México; rvivas@tunku.uady.mx químicos tales como organofosforados, piretroides, amidinas, fenilpirazolonas y lactonas macrocíclicas (LM) (FAO 1987, Rodríguez-Vivas y col 2005). Sin embargo, a través del tiempo, el uso irracional de estos químicos ha generado el desarrollo de resistencia a los diferentes ixodicidas (Fragoso y col 1999, Rodríguez-Vivas y col 2006 ${ }^{\mathrm{a}, \mathrm{b}}$ ), lo que ha propiciado la búsqueda de métodos alternativos de control de esta plaga en la industria bovina, tales como la selección de razas resistentes, control biológico (hongos entomopatógenos, depredadores y plantas), uso de vacunas (Jongejan y Uilenberg 1994, De la Fuente y col 1999, Kaaya y Hassan 2000, Willadsen 2001, Tedonkeng y col 2005) y el uso de lactonas macrocíclicas (LM) (Lanusse y col 1997). Las LM son endectocidas eficientes en el control de endo y ectoparásitos (Lanusse y col 1997). Las avermectinas (ejemplos: ivermectina, IVM; doramectina, DRM; eprinomectina, EPM; abamectina, ABM) y milbemicinas (ejemplo: moxidectina, MXD) son activas para el control de nematodos y artrópodos a dosis bajas en la mayoría de los animales domésticos (Sumano y Ocampo 2006). Se absorben por todas las vías debido a su alta liposolubilidad y se distribuyen ampliamente en los tejidos, tales como la luz intestinal, grasa y piel (Entrocasso y col 1996, Sumano y Ocampo 2006). Las LM producen su efecto antiparasitario al incrementar la permeabilidad de la membrana celular por los iones de cloro $\left(\mathrm{Cl}^{-}\right)$, con la consecuente hiperpolarización y parálisis de la musculatura faríngea y somática de los parásitos (Mudd y Parker 1995, Lifschitz y col 2002). El objetivo de la presente revisión 
es presentar información relevante de libros, tesis, congresos, seminarios y revistas con comité editorial sobre el uso de las principales LM (IVM, DRM, MXD, EPM, ABM) para el control de la garrapata $R$. (B.) microplus en el ganado bovino.

\section{LACTONAS MACROCÍCLICAS}

\section{FAMILIAS Y MECANISMOS DE ACCIÓN}

Las LM pertenecen a dos grandes familias según sea el actinomiceto de cuya fermentación provienen: avermectinas y milbemicinas (figura 1).

La compleja estructura química de estos fármacos corresponde a una lactona macrocíclica de 16 miembros similar a la de los antibióticos macrólidos (pero sin efecto bacteriano), unida a un grupo benzofurano $\left(\mathrm{C}_{2}\right.$ a $\left.\mathrm{C}_{8}\right)$ y a un anillo espiroquetal $\left(\mathrm{C}_{17} \mathrm{a} \mathrm{C}_{25}\right)$. Son moléculas de gran tamaño con peso molecular entre $600 \mathrm{kDa}$ (milbemicinas) y $800 \mathrm{kDa}$ (avermectinas) (Lifschitz y col 2002).

Las LM comparten algunas propiedades fisicoquímicas generales; pequeñas diferencias en la estructura química entre avermectinas y milbemicinas o aun dentro de las avermectinas determinan cambios en el comportamiento farmacocinético, lo cual repercute sobre la eficacia y la persistencia antiparasitaria de las mismas (Lifschitz y col 2002). En parásitos susceptibles, la IVM se une a un receptor de alta afinidad, lo que provoca un incremento en la permeabilidad de los iones $\mathrm{Cl}^{-}$, con el consiguiente desprendimiento del parásito por una parálisis flácida.

La identificación del receptor específico al cual se unen las LM ha sido objeto de controversia. Los primeros estudios describieron que la IVM producía una liberación de ácido gama-aminobutírico (GABA) desde los sinaptosomas del

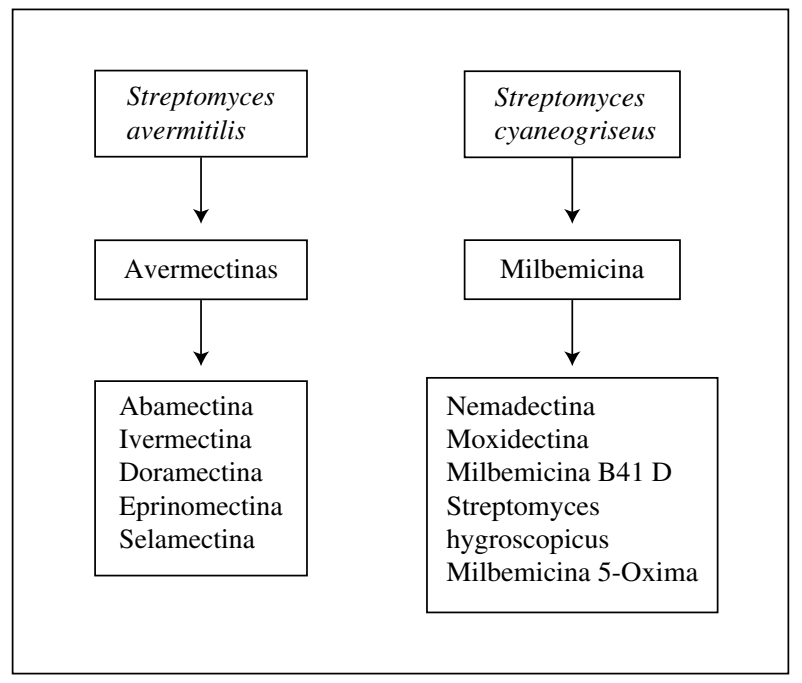

Figura 1. Origen y clasificación de las lactonas macrocíclicas: avermectinas y milbemicinas (Tomado de Lifschitz y col 2002).

Origen and classification of macrocyclic lactones: avermectins and milbemycins (From Lifschitz et al 2002). cerebro de rata, así como una modulación de los receptores gabérgicos que aumentaba la afinidad por el neurotransmisor. Por otro lado, dependiendo de las concentraciones de IVM a las que se exponen los parásitos, la entrada de $\mathrm{Cl}^{-}$podría o no estar mediada por un mecanismo gabérgico. Los datos actuales sugieren que la acción parasiticida de la avermectinas y milbemicinas viene dada por la interacción de las mismas con los canales de $\mathrm{Cl}^{-}$ligados a un receptor de glutamato en el parásito diana, lo cual daría lugar al fenómeno de hiperpolarización descrito (Mudd y Parker 1995, Lifschitz y col 2002).

Utilizando el nematodo de vida libre Canorhabditis elegans como modelo, se han podido caracterizar las subunidades de este receptor, observándose que a bajas concentraciones, las avermectinas potencian la acción del glutamato y a elevadas concentraciones producen directamente la apertura del canal de $\mathrm{Cl}^{-}$. Este receptor se ha localizado en la faringe de C. elegans y Ascaris summ y la estimulación del mismo inhibe la musculatura faríngea necesaria para la alimentación del parásito (Lifschitz y col 2002).

La información disponible hasta el momento sugiere que las milbemicinas comparten mecanismo de acción con las avermectinas, aunque no se descarta que existan diferencias farmacodinámicas muy sutiles entre ambos grupos (Lifschitz y col 2002).

La falta de actividad de las avermectinas y milbemicinas sobre trematodos y cestodos se debe a la ausencia, o al menos a una menor trascendencia, de la transmisión mediada por ese tipo de canales de $\mathrm{Cl}^{-}$en la coordinación neuromuscular de estos parásitos en comparación con nematodos o artrópodos (Sumano y Ocampo 2006).

\section{IVERMECTINA}

Descripción. La IVM es el resultado de la fermentación bacteriana del Streptomyces avermitilis, obtenido por primera vez en el año 1979. Es un fármaco muy liposoluble y poco hidrosoluble, por lo que se puede aplicar por todas las vías, siendo las más recomendadas la SC, intramuscular (IM) y tópica (“pour-on”) (Lifschitz y col 2002).

Composición química. Es un análogo semisintético de la abamectina, conteniendo un $80 \%$ de 22,23-dihidroavermectina B1a y no más de un $20 \%$ de 22,23, dihidroavermectina B1b (Lifschitz y col 2002).

Farmacocinética. La IVM es la LM de mayor difusión y utilización en las diferentes especies de animales en todo el mundo; por lo tanto, es la molécula que más se ha estudiado con respecto a sus características farmacodinámicas y farmacocinéticas. Las propiedades fisicoquímicas de la IVM incluyen un alto peso molecular y una elevada lipofilicidad, las que le confieren características farmacocinéticas de un alto volumen de distribución, con una gran afinidad por la grasa corporal y prolongada persistencia de 
sus concentraciones en el organismo (Sumano y Ocampo 2006).

El tiempo de liberación de la IVM es de sólo siete días después del tratamiento SC en bovinos. En un estudio se encontró que la IVM-3,15\% (0,63 mg/kg pv) inoculada a bovinos permanece en concentraciones plasmáticas $>2 \mathrm{ng} / \mathrm{ml}^{-1}$ a los 50 días PT (Lifschitz y col 2007). Los parámetros farmacocinéticos promedios de la IVM obtenidos en plasma después de ser administrados por vía $\mathrm{SC}$ a razón de $0,2 \mathrm{mg} / \mathrm{kg}$ pv en bovinos se presentan en el cuadro 1.

Presentación y dosificación. Las presentaciones comerciales que tiene la IVM son en solución oral, tópica e inyectable en el ganado bovino. La solución oral e inyectable son formulaciones acuosas, que contienen propilenglicol y solubilizantes, mientras que la tópica tiene únicamente el vehículo (McKellar y Benchaoui 1996). La dosis para la vía oral e inyectable es de $0,2 \mathrm{mg} / \mathrm{kg}$ pv. Sin embargo, actualmente se dispone de IVM de larga acción al 3,15\%, que se aplica vía SC a dosis de $0,63 \mathrm{mg} / \mathrm{kg}$ pv (Bridi y col 2001).

Seguridad y tiempo de retiro en el ganado bovino. El uso de IVM en el ganado bovino es seguro. Dosis de $4 \mathrm{mg} / \mathrm{kg}$ pv producen ataxia que se atribuyen a acciones sobre el sistema nervioso central. En general y con alguna variación según los diferentes países, la carne de animales tratados no debe destinarse al consumo humano durante períodos de 35-45 días (Lifschitz y col 2002).

Cuadro 1. Valores farmacocinéticos promedios obtenidos para la IVM, DRM y MXD en plasma después de ser administradas por vía $\mathrm{SC}$ a razón de $0,2 \mathrm{mg} / \mathrm{kg}$ pv en bovinos (Tomado de Lanusse y col 1997).

Mean pharmacokinetic values in plasma of IVM, DRM and MXD after a SC injection at $0.2 \mathrm{mg} / \mathrm{kg}$ in bovines (From Lanusse et al. 1997).

Parámetros cinéticos

Ivermectina Doramectina Moxidectina

\begin{tabular}{lrrr}
\hline T_ab (días) & 39,20 & 56,40 & 1,32 \\
$\mathrm{C}_{\text {max }}$ (ng/mL) & 42,80 & 37,50 & 39,40 \\
$\mathrm{~T}_{\max }$ (días) & 4,00 & 6,00 & 0,32 \\
$\mathrm{ABC}_{\text {total }}$ (ng.d/mL) & 459,00 & 627,00 & 217,00 \\
$\mathrm{TMR}$ (días) & 7,35 & 9,09 & 14,60 \\
$\mathrm{~V}_{\mathrm{dss}} / \mathrm{F}(\mathrm{L} / \mathrm{kg})$ & 3,35 & 2,92 & 13,60 \\
$\mathrm{Cl}_{\mathrm{B}} / \mathrm{F}(\mathrm{mL} / \mathrm{d} / \mathrm{kg}$ & 457,00 & 322,00 & 938,00 \\
\hline
\end{tabular}

T_ab: Tiempo medio de absorción; $\mathrm{C}_{\max }$ : Máxima concentración plasmática; $\mathrm{T}_{\max }$ : Tiempo en que se alcanza la $\mathrm{C}_{\max }: \mathrm{ABC}_{\text {total }}$ : área bajo la curva de concentración frente a tiempo extrapolada al infinito; TMR: Tiempo medio de residencia; $\mathrm{V}_{\mathrm{dss}} / \mathrm{F}$ : Volumen de distribución en el estado estacionario; $\mathrm{Cl}_{\mathrm{B}} / \mathrm{F}$ : Aclaramiento corporal total. $\mathrm{V}_{\mathrm{dss}}$ y $\mathrm{Cl}$ representan sus verdaderos valores en relación a la fracción absorbida del fármaco.
Seguridad ambiental. Las elevadas concentraciones de IVM que se eliminan por las heces mantienen su actividad biológica y ejercen su poder insecticida sobre un gran número de especies de dípteros y coleópteros que colonizan la materia fecal de los bovinos. Nichols y col (2008) mencionan que la IVM produce la muerte de varias especies de escarabajos coprófagos (paracópridos, telecópridos, endocópridos) que utilizan las heces de los bovinos para su anidación, reproducción y alimentación. La principal función de estos coleópteros radica en la incorporación de las heces al suelo al brindar servicios ecológicos de gran valor en los ecosistemas de pastizales, ya que la producción forrajera depende estrechamente del reciclaje de la materia orgánica producida y de la cantidad de elementos minerales disponibles (Suárez y col 2009).

La IVM se encuentra fuertemente unida a las partículas de heces bovinas, y sólo en una mínima proporción es arrastrada por el agua. La IVM tiene una vida media variable en mezclas de materia fecal y suelo, que va desde los 14 días en los meses de verano hasta los 240 días a $22^{\circ} \mathrm{C}$ en condiciones de laboratorio (Lifschitz y col 2002). Wardhaugh y Mahon (1998) reportan que los residuos de IVM en heces de bovinos tratados previnieron el desarrollo de Musca vettustissima, Musca domestica y Haematobia irritans durante 35 días en el ambiente. Asimismo, Guglielmone y col (1998) reportaron una eficacia de la IVM contra fases adultas de $H$. irritans en aproximadamente dos semanas.

\section{DORAMECTINA}

Descripción. La DRM se obtiene de Streptomyces avermitilis y es una avermectina de estructura y espectro similares a la IVM; sin embargo, tiene algunas diferencias que le confieren disponibilidad plasmática por un período más prolongado que la IVM (Sumano y Ocampo 2006).

Composición química. Es una avermectina de estructura y espectro similares a la ivermectina. Su nombre químico es 25-ciclohexil-5-O-demetil-25-(1-metilpropil)-avermectina A1a (Lifschitz y col 2002).

Farmacocinética. La DRM cuando se administra por vía SC tiene buena disponibilidad y eficacia contra nematodos y ectoparásitos. Se concentra más que otras ivermectinas en la luz intestinal. $\mathrm{Al}$ administrar en bovinos una dosis de $0,2 \mathrm{mg} / \mathrm{kg}$ pv por vía IM, se logra una concentración de $33,1 \mathrm{ng} / \mathrm{ml}$, y por vía $\mathrm{SC}$ se logran concentraciones variables que van de 27,8 a 37,5 ng/ml (Lanusse y col 1997). Cuando se administra por vía IM se requiere un tiempo de cuatro días para alcanzar las concentraciones plasmáticas máximas y de seis días cuando se administra por vía SC (Lanusse y col 1997). La mayor parte del fármaco administrado por vía IM o SC no se metaboliza, y de tres a 21 días después de la administración se encuentra DRM sin cambios en un 58-70\% como residuo en tejidos, con 91\% en grasa. Después de 14 días de su administración 
por vía SC se encuentra $87 \%$ en heces y sólo $1 \%$ en orina (Lifschitz y col 2007). Los parámetros farmacocinéticos promedios de la DRM obtenidos en plasma después de ser administrados por vía SC a razón de $0,2 \mathrm{mg} / \mathrm{kg}$ pv en bovinos se presentan en el cuadro 1 .

Presentación y dosificación. Las presentaciones comerciales que tiene la DRM en el ganado bovino son tópicas e inyectables. La dosis para la vía inyectable es de $0,2 \mathrm{mg}$ / kg pv (Lifschitz y col 2002).

Seguridad y tiempo de retiro en el ganado bovino. El uso de DRM en el ganado bovino es seguro. La DRM se tolera razonablemente; una dosis por vía $\mathrm{SC}$ a razón de $0,6 \mathrm{mg} / \mathrm{kg}$ pv aplicada entre los días 12 y 55 de gestación no provoca efectos adversos en desarrollo embrionario, mantenimiento de la gestación, parto o supervivencia del producto, y en los machos no afecta la calidad del semen. Al igual que la IVM-1\%, la carne de animales tratados no debe destinarse al consumo humano durante períodos de 35-45 días (Lifschitz y col 2002).

Seguridad ambiental. En un estudio realizado por Suárez y col (2009) se aplicaron MXD (SC, “pour-on”) y DRM (SC) a bovinos y se evaluó el efecto de la excreción de los fármacos en heces sobre la sobrevivencia de artrópodos benéficos. En este estudio se concluyó que la DRM tiene mayor efecto adverso sobre artrópodos que la MXD. Asimismo, Anziani y col (2001) evaluaron la actividad de la DRM en bovinos tratados contra nes de campo de $H$. irritans y observaron que en las heces no emergían adultos durante 35 días después del tratamiento.

\section{MOXIDECTINA}

Descripción. La MXD es una LM sintetizada en 1990 en Japón, es muy activa contra nematodos y artrópodos a dosis bajas en la mayoría de los animales domésticos (Sumano y Ocampo 2006). Es un fármaco que puede usarse con 4 a 5 veces la dosis terapéutica en ovinos sin observarse reacciones negativas. En bovinos no se han observado reacciones negativas incluso con dosis 10 veces mayores a las recomendadas (Sumano y Ocampo 2006). Las dosis terapéuticas usadas son 0,2 a $1,0 \mathrm{mg} / \mathrm{kg}$ pv con resultados satisfactorios. Su espectro abarca fases adultas e inmaduras, asimismo reduce la capacidad reproductiva de las garrapatas (Caracostantogolo y col 1993, Sibson 1994).

Composición química. El nombre químico es [6R,23E,25S(E) 5-0-desmetil-28-dioxi-25-(1-butenil)-6,28 epoxi-23(metoximino) milbemicina $\mathrm{B}$; cuenta con un peso molecular de $639,8 \mathrm{kDa}$ y su fórmula condensada es $\mathrm{C}_{37} \mathrm{H}_{53} \mathrm{NO}_{8}$. Es un derivado semisintético de la nemadectina, que es un anillo lactona macrocíclico producido por fermentación de Streptomyces cyanogriseus. La estructura química se relaciona con las milbemicinas y avermectinas, con las que comparte además de la similitud de su molécula el tipo de absorción y la farmacocinética (Sumano y Ocampo 2006).

Farmacocinética. El fármaco se absorbe por todas las vías debido a que es muy liposoluble; se considera más lipofílica e hidrofóbica que la IVM, se distribuye ampliamente en los tejidos, pero por su ciclo enterohepático se acumula sobre todo en la luz intestinal, grasa y piel, lo que permite su uso como ixodicida con buenos resultados. La vida media en bovinos es de 9 a 11 días en promedio, con un efecto residual de tres meses, lo cual permite programar con mayor intervalo el calendario de desparasitación (Entrocasso y col 1996).

Lifschitz y col (1999) utilizando MXD-1\% a dosis de $0,2 \mathrm{mg} / \mathrm{kg}$ pv encontraron que la concentración máxima de MXD en plasma se alcanzó al día 1 PT $\left(84,2 \mathrm{ng} / \mathrm{ml}^{-1}\right)$ y durante los primeros ocho días PT la concentración de la droga en plasma fue de $9 \mathrm{ng} / \mathrm{g}^{-1}$. La concentración de la MXD en la piel declinó gradualmente con el paso del tiempo, aunque en otros tejidos fue detectada hasta por 58 días (>0,2 ng/ ml-1). Estas concentraciones altas de MXD y su presencia prolongada en la piel la hacen ser una droga eficaz contra diferentes ectoparásitos en rumiantes.

En un estudio realizado en Francia por Dupuy y col (2007) utilizando MXD-10\% a dosis de $1 \mathrm{mg} / \mathrm{kg}$ pv en bovinos se detectó por primera vez MXD en plasma una hora PT $\left(2,00 \pm 1,52 \mathrm{ng} / \mathrm{ml}^{-1}\right)$ y dos días PT en pelo a concentración de $\left(446,4 \pm 193,26 \mathrm{ng} / \mathrm{g}^{-1}\right)$. El tiempo en que se encontraron concentraciones en plasma y pelo por encima de $2 \mathrm{ng} / \mathrm{ml}^{-1}$ o $2 \mathrm{ng} / \mathrm{g}^{-1}$ fue de 120 y 100 días, respectivamente. Los parámetros farmacocinéticos promedios de la MXD obtenidos en plasma después de ser administrados por vía SC a razón de $0,2 \mathrm{mg} / \mathrm{kg}$ pv en bovinos se presentan en el cuadro 1 .

Presentación y dosificación. Las presentaciones comerciales que tiene la MXD son en solución oral, inyectable y epicutánea. La solución oral e inyectable son formulaciones acuosas, que contienen propilenglicol y solubilizantes; mientras que la forma epicutánea tiene únicamente un vehículo (Aguilar-Tipacamú y Rodríguez-Vivas 2002). La dosis para la vía oral e inyectable es de $0,2 \mathrm{mg} / \mathrm{kg}$ pv y la presentación tópica es utilizada a dosis de $0,5 \mathrm{mg} / \mathrm{pv}$ (Geurden y col 2004). Sin embargo, actualmente se dispone de una MXD de larga acción al 10\%, que se aplica vía SC dosis de $1 \mathrm{mg} / \mathrm{kg} \mathrm{pv}$.

Seguridad y tiempo de retiro. No ocurre muerte de bovinos cuando son inyectados por vía SC hasta 10 veces la dosis recomendada $(2,0 \mathrm{mg} / \mathrm{kg} \mathrm{pv})$. Cuando fue aplicada tres veces la dosis recomendada $(0,6 \mathrm{mg} / \mathrm{kg} \mathrm{pv})$, no se observaron efectos sobre la reproducción de toros, vacas gestantes y vaquillas cuando fue inyectada en cada trimestre de la gestación (Rae y col 1994). Cuando la MXD se administra por vía $\mathrm{SC}$, es posible detectar el fármaco y siete de sus metabolitos en diferentes tejidos, heces y bilis. Si se maneja 
un tiempo de retiro de 30 días se pueden encontrar hasta 2 ppb en algunos tejidos (Sumano y Ocampo 2006).

Seguridad ambiental. El estiércol de animales tratados con MXD produce menor efecto adverso que la DRM e IVM en contra de artrópodos benéficos que se encuentran en las heces de bovinos tales como los escarabajos coprófagos (ejemplo: Sulcophanaeus, Digitonthophagus o Canthidium) (Suárez y col 2009). Aunque los residuos en heces de bovinos tratados con MXD inyectable no tuvieron efectos significativos sobre larvas de M. vettustissima y M. domestica (Wardhaugh y col 1996), otros experimentos muestran que la MXD en las heces de bovinos tiene actividad contra las fases inmaduras de $H$. irritans (Suárez y col 2009).

\section{EPRINOMECTINA}

Descripción. La EPM es una molécula que se obtiene a partir de la fermentación del Streptomyces avermitilis (Blagburn y Lindsay 2001). La sustitución de un grupo epi-acetilamino en la posición 4" es la diferencia con las otras avermectinas $B_{1}$ (Lifschitz y col 2002).

Composición química. Es un miembro del grupo de las avermectinas biosintéticas. También se le conoce como MK-397. Es un sólido cristalino cuyo nombre químico es (4"R)-4" -epi-(acetilamino)-4"-desoxiavermectina $B_{1}$ (Blagburn y Lindsay 2001, Sumano y Ocampo 2006).

Farmacocinética. La EPM ha sido desarrollada para su administración en forma epicutánea, conservando su actividad contra endo y ectoparásitos. Debido a que son compuestos muy liposolubles su distribución es muy buena, presentando un elevado porcentaje de unión a proteínas plasmáticas, concentrándose principalmente en grasa e hígado. Su absorción, una vez colocada sobre el animal, comienza inmediatamente y se mantiene en un buen nivel hasta 10 días después de la administración. Es muy poco metabolizada y como las otras avermectinas se elimina en forma activa por heces. Lo más interesante de este nuevo compuesto es que su uso en vacas lecheras no requiere tiempo de retiro en leche (Dupuy y col 2001). Esto se debe a una combinación de factores: por un lado, la EPM presenta un perfil residual bajo, por otro, su límite máximo de residuos es más elevado que las otras avermectinas. Su metabolización se lleva a cabo en el hígado y se elimina principalmente en heces; menos del 1\% se elimina en la orina (Sumano y Ocampo 2006).

Presentación y dosificación. La presentación comercial que tiene la EPM es epicutánea en el ganado bovino. La dosis por la vía tópica es de $0,5 \mathrm{mg} / \mathrm{kg}$ pv (Lifschitz y col 2002, Sumano y Ocampo 2006).

Seguridad y tiempo de retiro en el ganado bovino. Estudios realizados en bovinos y cerdos, incluyendo hembras en diversos estados de gestación y machos reproductores, han demostrado un amplio margen de seguridad cuando es administrado a la dosis recomendada (Lifschitz y col 2002). No debe administrarse por vía oral o intravenosa. Los bovinos toleran por vía percutánea dosis hasta tres veces mayores que la indicada. Una de las ventajas que tiene la EPM comparada con otras LM es que al aplicarla $(0,5 \mathrm{mg} /$ $\mathrm{kg}$ pv) en vacas lactantes los residuos de la droga en la leche son bajos, por lo que se puede usar para el consumo humano (Alvinerie y col 1999, Blagburn y Lindsay 2001, Lifschitz y col 2002). Sin embargo, en Nueva Zelanda se recomiendan hasta 14 días de retiro con las mismas indicaciones de aplicación (Sumano y Ocampo 2006).

Seguridad ambiental. Las avermectinas incluyendo la EPM tienen una semivida variable en mezclas de materia fecal y suelo, que va desde los 14 días en los meses de verano hasta los 240 días a $22{ }^{\circ} \mathrm{C}$ en condiciones de laboratorio (Lifschitz y col 2002). En la actualidad no existen estudios del efecto de la EPM sobre poblaciones de artrópodos a nivel de campo.

\section{ABAMECTINA}

Descripción. También se le denomina avermectina $\mathrm{B}_{1}$ (Mrozik 1994). Se encuentra en forma de cristales blancoamarillentos y es inodora. Es un producto natural que se obtiene también de la fermentación de Streptomyces avermitilis, del cual se forman dos homólogos, la avermectina $\mathrm{B}_{1}$ y la $\mathrm{B}_{2}$, que se diferencian por un grupo metilo. El compuesto es similar a la ivermectina, de la que solamente difiere por la presencia de una doble ligadura en los carbonos 22 y 23 (Mrozik 1994, Lifschitz y col 2002).

Composición química. Su nombre químico es 5-O-dimetilavermectina A1a y 5-O-dimetil-25-des (1-metilpropil)-25-(1-metiletil) avermectina $\mathrm{A}_{1 \mathrm{a}}$ (4:1); avermectina $\mathrm{B}_{1}$ a/b. Su fórmula molecular es $\mathrm{C}_{48} \mathrm{H}_{72} \mathrm{O}_{14}$; $\mathrm{C}_{47} \mathrm{H}_{70} \mathrm{O}_{14}$ (Sumano y Ocampo 2006).

Farmacocinética. Los procesos de absorción están relacionados con la vía de administración del fármaco. Su distribución es muy amplia; se acumula principalmente en hígado y tejido adiposo, pero llega con facilidad a la piel. Los procesos de metabolismo se sujetan a la hidroxilación del producto, y la excreción se realiza principalmente por las heces, la orina y una mínima proporción en leche. Es importante señalar que si se acelera el tránsito intestinal, ésta y otras avermectinas serán menos biodisponibles (Sumano y Ocampo 2006).

Presentación y dosificación. En todos los casos se administra una sola dosis y se pueden utilizar, según la presentación, las vías oral, tópica y SC (Mrozik 1994, Lifschitz y col 2002); en esta última se informa ligera inflamación a los 10 días. La ABM se ha utilizado con bastante éxito en el control de 
las parasitosis con nematodos gastrointestinales del ganado en dosis de 0,2 mg/kg pv por vía subcutánea (Sumano y Ocampo 2006).

Seguridad y tiempo de retiro en bovinos. No se recomienda administrarla en bovinos menores de cuatro meses de edad. La aplicación parenteral ha causado lesiones sépticas por Clostridium sp y miositis, incluso fatales. Los ensayos en animales de laboratorio muestran que la abamectina $\mathrm{B}_{1 \mathrm{a}}$ no se absorbe por vía oral en mamíferos y se elimina por completo en las heces en dos días. Para bovinos el tiempo de retiro es de 30-45 días, dependiendo de la presentación farmacéutica que se haya aplicado, en cambio para ovinos es de 14 a 21 días (Sumano y Ocampo 2006). Palma y col (2006) realizaron un estudio para determinar los niveles de residuos de ABM y triclobendazol (TCBZ) en tejidos comestibles de bovinos tratados con la formulación comercial que contiene la asociación de ambos fármacos para administración vía oral en bovinos. Se utilizaron 16 bovinos con un peso promedio de $232 \pm 37,5 \mathrm{~kg}$, los cuales fueron tratados con la asociación ABM-TCBZ a una dosis de $0,2 \mathrm{mg}$ de ABM/kg pv y $10 \mathrm{~kg}$ de TCBZ/kg pv. Los animales fueron sacrificados en grupos de tres a cuatro animales por semana desde el día siete hasta el día 42 posterior al tratamiento. No se registraron concentraciones detectables de ABM en grasa, riñón y músculo a los 14 , 28 y 42 días postratamiento. Las mayores concentraciones residuales de $\mathrm{ABM}$ fueron observadas en las muestras de hígado $(4,02 \mathrm{ng} / \mathrm{g})$, las que persisten por un período de 14 días. Estos hallazgos se pueden atribuir a la alta lipofilicidad de ABM que le confiere mayor afinidad por el tejido hepático.

Seguridad ambiental. La ABM se degrada rápidamente en la superficie del suelo, donde sufre fotodegradación, y se han informado vidas medias desde 8 h hasta un día. Cuando se aplica en la superficie del suelo y no se protege del sol, la vida media en el suelo es de aproximadamente una semana. Bajo la oscuridad y en condiciones aeróbicas, ese valor es de dos semanas a dos meses. La ABM se degrada rápidamente en el agua. Su vida media en agua superficial estancada es de cuatro días, y en sedimento estancado, de dos a cuatro semanas. La ABM sufre una rápida fotodegradación y tiene vida media en el agua de $12 \mathrm{~h}$. Las plantas no absorben la ABM a partir del suelo. El fármaco se degrada rápidamente cuando está presente en forma de una capa delgada (superficie de las hojas); en un laboratorio bajo estas condiciones y en presencia de luz, su vida media fue de 4 a 6 h (Sumano y Ocampo 2006).

La ABM es atóxica para las aves, la DL50 en codornices es $>2000 \mathrm{mg} / \mathrm{kg}$. En organismos acuáticos la ABM es altamente tóxica para los peces y extremadamente tóxica para invertebrados acuáticos. Aunque la ABM es altamente tóxica para los organismos acuáticos, las concentraciones encontradas en aguas superficiales adyacentes a áreas tratadas suelen ser bajas (Sumano y Ocampo 2006).

\section{EFICACIA DE LAS LACTONAS MACROCÍCLICAS PARA EL CONTROL DE GARRAPATAS EN BOVINOS}

En el cuadro 2 se presentan las eficacias, vías de aplicación y persistencia de IVM, DRM, MXD, EPM y ABM a diferentes dosificaciones contra la garrapata $R$. (B.) microplus.

Por un lado, en otros estudios se compararon IVM contra MXD (0,2 mg/kg pv) por vía $\mathrm{SC}$, obteniendo una eficacia del 99\% la IVM y del 99,1\% la MXD sobre la reducción de hembras que alcanzaron la repleción; y sobre la reducción de la oviposición de hembras adultas que alcanzaron la repleción. Por otra parte, también se evaluó la eficacia de ambas LM contra larvas de $R$. (B.) microplus en bovinos infestados en condiciones controladas a las semanas uno y cuatro PT, reportándose eficacias para IVM del $82,4 \%$ (1 sem) a 0,0\% (4 sem) y para MXD del $92,4 \%$ (1 sem) a 19,5\% (4 sem) (McKellar y Benchaoui 1996, Davey y col 2005).

Bridi y col (1992) evaluaron la eficacia de la ABM para el control de $R$. (B.) microplus en bovinos usando $0,1,0,2$ y $0,3 \mathrm{mg} / \mathrm{kg}$ pv vía subcutánea y encontraron una reducción en el índice de reproducción de las garrapatas de 90, 94 y 96\% respectivamente, no observándose diferencia significativa en las distintas dosis evaluadas.

\section{RESISTENCIA DE RHIPICEPHALUS (BOOPHILUS) MICROPLUS A LAS LACTONAS MACROCÍCLICAS}

La alta liposolubilidad de las LM le confiere características farmacocinéticas de alto volumen de distribución, con una gran afinidad por la grasa corporal. Debido a estas características y a la disponibilidad en el mercado de LM de larga acción (IVM-3,15\%, IVM-4\%, MXD-10\%), estos fármacos al ser aplicados a los bovinos persisten en sangre, grasa y piel por períodos prolongados, exponiendo a las garrapatas a concentraciones letales o subletales que pudieran generar en el futuro poblaciones de garrapatas resistentes.

La resistencia a LM ha sido mundialmente descrita en algunas especies de nematodos. Casos de nematodos gastrointestinales resistentes a la IVM han sido reportados en Argentina, Brasil, Venezuela, Estados Unidos, Nigeria, India, Bangladesh, Australia y Nueva Zelanda (Fashanu y Fagbemi 2003, Soutello y col 2007). Sin embargo, sólo se han reportado garrapatas $R$. (B.) microplus resistentes a la IVM en la ganadería bovina de Brasil (Martins y Furlong 2001, Klafke y col 2006). Debido al uso cada vez más frecuente de las LM en los ranchos bovinos para el control de endo- y ectoparásitos en México (Soberanes 2007) y algunos reportes de ganaderos sobre la baja eficacia de las LM cuando son aplicadas, es necesario realizar monitoreos constantes de la susceptibilidad de $R$. (B.) microplus a las LM para poder detectar tempranamente casos de resistencia que permitan establecer oportunamente las medidas de control pertinentes. 
Cuadro 2. Eficacia y persistencia de las lactonas macrocíclicas sobre Rhipicephalus (Boophilus) microplus.

Efficacy and persistence of macrocyclic lactones on Rhipicephalus (Boophilus) microplus.

\begin{tabular}{|c|c|c|c|c|c|}
\hline Principio activo & $\begin{array}{c}\text { Dosis } \\
(\mathrm{mg} / \mathrm{kg} / \mathrm{pv})\end{array}$ & Vía de aplicación & Eficacia $(\%)$ & $\begin{array}{l}\text { Persistencia } \\
\text { (días PT) }\end{array}$ & Autor \\
\hline Ivermectina $0,5 \%$ & 0,5 & Tópica & 85 & 23 & Davey and George 2002 \\
\hline Ivermectina $0,5 \%$ & $\begin{array}{l}0,2 \\
0,5 \\
1,0\end{array}$ & $\begin{array}{l}\text { Tópica } \\
\text { Tópica } \\
\text { Tópica }\end{array}$ & $\begin{array}{l}50 \\
85 \\
91\end{array}$ & $\begin{array}{l}35 \\
35 \\
35\end{array}$ & Cramer y col 1988 \\
\hline Ivermectina $1 \%$ & 0,2 & Subcutánea & 99 & 28 & McKellar y Benchaoui 1996, Davey y col 2005 \\
\hline Ivermectina $1 \%$ & 0,2 & Subcutánea & 80 & 35 & Cramer y col 1988 \\
\hline Ivermectina $3,15 \%$ & 0,63 & Subcutánea & 87 & 63 & Borges y col 2008 \\
\hline Ivermectina $3,15 \%$ & 0,63 & Subcutánea & 95 & 56 & Arieta-Román 2008 \\
\hline Moxidectina $0,5 \%$ & 0,5 & Tópica & 79 & 23 & Davey y George 2002 \\
\hline Moxidectina $1 \%$ & 0,2 & Subcutánea & $95-99$ & 28 & $\begin{array}{l}\text { Aguilar-Tipacamu y Rodríguez-Vivas } 2003 \text {, } \\
\text { Remington y col 1997, Coracostantogolo } 1993\end{array}$ \\
\hline Moxidectina $10 \%$ & 1,0 & Subcutánea & $>95$ & 70 & CNACSA 2007, Arieta-Román y col 2008 \\
\hline Doramectina $0,5 \%$ & 0,5 & Tópica & 89 & 21 & George y Davey 2004 \\
\hline Doramectina $1 \%$ & 0,2 & Subcutánea & 94 & 21 & George y Davey 2004 \\
\hline Doramectina $1 \%$ & 0,2 & Subcutánea & $91-99$ & 28 & Muniz y col 1995 \\
\hline Eprinomectina $0,5 \%$ & 0,5 & Tópica & 88 & 23 & Davey y George 2002 \\
\hline Eprinomectina $0,5 \%$ & $\begin{array}{l}0,5 \\
1,0\end{array}$ & $\begin{array}{l}\text { Tópica } \\
\text { Tópica }\end{array}$ & $\begin{array}{l}90 \\
98\end{array}$ & $\begin{array}{l}35 \\
35\end{array}$ & Aguirre y col 2005 \\
\hline $\begin{array}{l}\text { Ivermectina } 2,25 \% \\
+ \text { Abamectina } 1,25 \%\end{array}$ & $0,45+0,25$ & Subcutánea & 91 & 63 & Borges y col 2008 \\
\hline Abamectina $1 \%$ & 0,2 & Subcutánea & 80 & 21 & Pereira 2009 \\
\hline
\end{tabular}

\section{CONCLUSIONES}

Se concluye que las LM por su alta liposolubilidad se distribuyen ampliamente en los tejidos, siendo eficaces para el control de $R$. (B.) microplus en el ganado bovino. La eficacia de la IVM, DRM, MXD, EPM y ABM de corta acción es similar (>90\% de eficacia a las cuatro semanas PT) para el control de fases adultas de $R$. (B.) microplus; sin embargo, existen en el mercado LM de larga acción que presentan eficacia $>95 \%$ con persistencia hasta 70 días PT. Los resultados de las investigaciones demuestran que las LM son una buena alternativa de control de garrapatas en el ganado bovino. Debido a la elevada persistencia de las LM (en especial las de larga acción) en los tejidos de bovinos tratados y al contacto constante de las garrapatas a estos fármacos podrían en el futuro promover la generación de poblaciones de garrapatas resistentes. Por tal motivo, el monitoreo constante de la susceptibilidad de $R$. (B.) microplus a las LM se hace necesario con la intención de detectar casos tempranos de resistencia y establecer los programas de control pertinentes.

\section{RESUMEN}

En esta revisión se describe en detalle la composición química, farmacocinética, presentación y dosis, seguridad y tiempo de retiro y la seguridad ambiental de los grupos que componen a las LM: avemectinas (ivermectina, doramectina, eprinomectina, abamectina) y milbemicinas (moxidectina). Además se presenta una discusión sobre su eficacia y el problema de resistencia de Rhipicephalus (Boophilus) microplus a las LM. Se concluye que las LM por su alta liposolubilidad se distribuyen ampliamente en los tejidos, siendo eficaces para el control de $R$. (B.) microplus en el ganado bovino. La eficacia de la ivermectina, doramectina, eprinomectina, abamectina y moxidectina de corta acción es similar ( $>90 \%$ de eficacia a las cuatro semanas postratamiento, PT) para el control de fases adultas de $R$. (B.) microplus; sin embargo, existen en el mercado LM de larga acción que presentan eficacia $>95 \%$ con persistencia hasta 70 días PT.

\section{REFERENCIAS}

Aguilar-Tipacamu G, RI Rodríguez-Vivas. 2002. Uso de la moxidectina para el tratamiento de los parásitos internos y externos de los animales. Rev Bioméd 13, 43-51.

Aguilar-Tipacamu G, RI Rodríguez-Vivas. 2003. Effect of moxidectin against natural infestation of the cattle tick Boophilus microplus (Acarina: Ixodidae) in the Mexican tropics. Vet Parasitol 111, 211-216.

Aguirre DH, AB Gaido, MM Cafrune, ME Castelli, AJ Mangold, AA Guglielmone. 2005. Eprinomectin pour-on for control of Boophilus microplus (Canestrini) ticks (Acari: Ixodidae) on cattle. Vet Parasitol 127, 157-163.

Alvinerie M, JF Sutra, P Galtier, C Mage. 1999. Pharmacokinetics of eprinomectin in plasma and milk following topical administration to lactating dairy cattle. Rev Vet Sci 67, 229-232.

Anziani OS, SG Flores, AA Guglielmone. 2001. Activity of injectable doramectin against Haematobia irritans in cattle. Rev Bras Parasitol Vet 9, 115-118. 
Arieta-Román RJ, RI Rodríguez-Vivas, JA Rosado-Aguilar, GT RamírezCruz, G Basto-Estrella. 2008. Moxidectina (10\%) e Ivermectina $(3,15 \%)$ : evaluación de su eficacia y persistencia contra infestaciones naturales de Rhipicephalus (Boophilus) microplus en bovinos del trópico mexicano. VI Seminario Internacional de Parasitología Animal, Boca del Río Veracruz, México.

Blagburn BL, DS Lindsay. 2001. Ectoparasiticides. In: Adams HR (ed). Veterinary Pharmacology and Therapeutics. $8^{\text {th }}$ ed. Blackwell Publishing, USA, Pp 1017-1039.

Borges FA, HC Silva, C Buzzulini, VE Soares, E Santos, GP Oliveira, AJ Costa. 2008. Endectocide activity of a new long-action formulation containing $2.25 \%$ ivermectin $+1.25 \%$ abamectin in cattle. Vet Parasitol 155, 299-307.

Bridi A, L Carvalho, L Cramer, S Gross, J Cruz, N Amaral. 1992. Efficacy of abamectin against the cattle tick Boophilus microplus Acarina, Ixodidae. Rev Brasil Parasitol Vet 1, 35-40.

Bridi A, L Carvalho, L Cramer, R Barrick. 2001. Efficacy of a long formulation of ivermectin against Psoroptes ovis (Hering 1838) on cattle. Vet Parasitol 97, 277-283.

Caracostantogolo J, CS Eddi, GM Bulman, ME Morley, A Noaco. 1993. Moxidectin: efficacy and dose titration in cattle experimentally infected with Boophilus microplus in Argentina. Proc. 14th Ibt. Conference WAAVP, Cambridge, England.

CNSCSA, Centro Nacional de Servicios de Constatación en Salud Animal. 2007. Evaluación de establo de la moxidectina al $10 \%$ para el control de Boophilus microplus. Prueba de constatación. SAGARPA-SENASICA, Jiutepec, Morelos, México.

Cramer LG, LA Carvalho, AA Brida, NK Amaral, RA Barrik. 1988. Efficacy of topically applied ivermectin agains Boophilus microplus (Canestrini, 1887) in cattle. Vet Parasitol 29, 341-349.

Davey RB, JE George. 2002. Efficacy of macrocyclic lactone endectocides against Boophilus microplus (Acari: Ixodidae) infested cattle using different pour-on application treatment regimes. J Med Entomol 39, 763-769.

Davey RB, JA Miller, JE George, RJ Miller. 2005. Therapeutic and persistent efficacy of a single injection treatment of ivermectin and moxidectin against Boophilus microplus (Acari: Ixodidae) on infested cattle. Exp Appl Acarol 35, 117-129.

De la Fuente J, Rodríguez M, Montero C. 1999. Vaccination against ticks (Boophilus microplus): the experience with the Bm86-based vaccine Gavac $^{\mathrm{TM}}$. Cuba. Gen Anal 15, 143-148.

Dupuy J, C Chartier, JF Sutra, M Alvinerie. 2001. Eprinomectin in dairy goats: dose influence on plasma levels and excretion in milk. Parasitol Res 87, 294-298.

Dupuy J, FJ Sutra, M Alvinerie. 2007. Pharmacokinetics assessment of moxidectin long-acting formulation in cattle. Vet Parasitol 147, 252-257.

Entrocasso D, D Parra, D Vottero, M Farías, LF Uribe, WG Ryan. 1996. Comparison of the persistent activity of ivermectin, abamectin, doramectin and moxidectin in cattle. Vet Rec 138, 91-92.

FAO, Food and Agriculture Organization of the United Nations. 1987. Control de las garrapatas y de las enfermedades que trasmiten. Manual práctico de campo, FAO 1, 5-20.

Fashanu SO, BO Fagbemi. 2003. A preliminary investigation of resistance to anthelmintics in strongyles of cattle in Shaki, Nigeria. Afr $J$ Biomed Res 6, 111-112.

Fragoso SH, EM Ortiz, V De Labra, NN Ortiz, M Rodríguez, M Redondo, J De la Fuente, PV Hernández. 1999. Evaluación de la vacuna contra la garrapata Bm86 (Gavac) para el control de Boophilus microplus. En: García VZ, Fragoso SH (eds) IV seminario Internacional de Parasitología control de la resistencia en garrapatas y moscas de importancia veterinaria y enfermedades que trasmiten. Puerto Vallarta, Jalisco, México, Pp 47-50.

George JE, RB Davey. 2004. Therapeutic and persistent efficacy of a single application of doramectin applied either as a pour-on or injection to cattle infested with Boophilus microplus (Acari: Ixodidae). J Med Entomol 41, 402-407.
Geurden T, E Claerebout, E Deroover, J Vercruysse. 2004. Evaluation of the hemoprophylactic efficacy of $10 \%$ long acting injectable moxidectin against gastrointestinal nematode infections in calves in Belgium. Vet Parasitol 120, 331-338.

Guglielmone AA, MM Volpogni SG Flores, GM Bulman, OS Anziani, JC Lamberti, OA Mancebo. 1998. Evaluación de una formulación de ivermectina al $1 \% \mathrm{p} / \mathrm{p}$ inyectable para el control de infestaciones naturales de bovinos por Haematobia irritans (Lineo, 1758) (Diptera: Muscidae) en Santa Fe (Argentina). Vet Argent 15, 170-175.

Jongejan E, G Uilenberg. 1994. Ticks and control methods. Sci Technol Integ Epizotiol 13, 1201-1226.

Kaaya GP, S Hassan. 2000. Entomogenous fungi as promising biopesticides for tick control. Exp Appl Acarol 24, 913-926.

Klafke GM, G Sabatini, T De Albuquerque, JR Martins, D Kemp, RJ Miller, T Schumaker. 2006. Larval immersion tests with ivermectin populations of the cattle tick Rhipicephalus (Boophilus) microplus (Acari: Ixodidae) from State of São Paulo, Brazil. Vet Parasitol 142, 386-390.

Lanusse C, A Lifschitz, G Virkel, L Alvarez, S Sánchez, JF Sutra, P Galtier, M Alvinerie. 1997. Comparative plasma disposition kinetics of ivermectin, moxidectin and doramectin in cattle. $J$ Vet Pharmacol Ther 20, 91-99.

Lifschitz A, G Virkel, P Imperiale, C Galtier, C Lanusse, M Alvinerie. 1999. Moxidectin in cattle: correlation between plasma and target tissues disposition kinetics. J Vet Pharmacol Ther 22, 266-273.

Lifschitz A, G Virkel, F Imperiale, A Pis, C Lanusse. 2002. Fármacos endectocidas: avermectinas y milbemicinas. En: Botana LM, Landoni F, Matín-Jiménez T (eds). Farmacología y Terapéutica Veterinaria. McGraw-Hill-Interamericana, Madrid, España, Pp 545-558.

Lifschitz A, G Virkel, M Ballent, J Sallovitz, F Imperiale, A Pis, C Lanusse. 2007. Ivermectin (3.15\%) long-acting formulations in cattle: absorption pattern and pharmacokinetic considerations. Vet Parasitol 147, 303-310.

Martins J, J Furlong. 2001. Avermectin resistance of the cattle tick Boophilus microplus in Brazil. Vet Rec 149, 64.

McKellar QA, H Benchaoui. 1996. Avermectins and milbemycins. J Vet Pharmacol Therap 19, 331-35.

Mrozik H. 1994. Advances in research and development of avermectins. In: Hedin PA, Menn JJ, Hollingworth RM (eds). ACS Symposium Series No. 551, Natural and Engineered Pest Management Agents, American Chemical Society, Washington DC, USA, Pp 54-73.

Mudd AJ, LD Parker. 1995. Use of the new molecule moxidectin for the control of parasites in sheep. Proceed Sheep Vet Soc 18, 139-143.

Muniz RA, F Hernández, O Lombardero, RC Leite, J Moreno, J Errecalde, LC Gonçalves. 1995. Efficacy of injectable doramectin against natural Boophilus microplus infestations in cattle. Am J Vet Res $56,460-463$.

Nichols E, S Spectora, J Louzadab, T Larsenc, S Amezquitad, ME Favilad. 2008. Ecological functions and ecosystem services provided by Scarabaeinae dung beetles. Biol Conserv 141, 1461-1474.

Palma C, C Godoy, M Arboix, R Pérez. 2006. Determinación de residuos de abamectina-triclabendazol en tejidos bovinos. Arch Med Vet 38, 265-271.

Pereira JR. 2009. The efficiency of avermectins (abamectin, doramectin and ivermectin) in the control of Boophilus microplus, in artificially infested bovines kept in field conditions. Vet Parasitol 162, 116-119.

Rae DO, RE Larsen, GT Wang. 1994. Safety assessment of moxidectin $1 \%$ injectable on reproductive performance in beef cows. Am J Vet Res 55, 251-253.

Remington B, P Kieran, R Coob, D Bodero. 1997. The application of moxidectin formulations for control of the cattle tick (Boophilus microplus) under Queensland field conditions. Aust Vet J 75, 588-591.

Rodríguez-Vivas RI, AF Quiñones, SH Fragoso. 2005. Epidemiología y control de la garrapata Boophilus en México. En: Rodríguez-Vivas, RI (ed) Enfermedades de importancia económica en producción animal. McGraw-Hill-UADY. México D.F., México, Pp 571-592. 
Rodríguez-Vivas RI, MA Alonso-Díaz, F Rodríguez-Arévalo, H FragosoSánchez, VM Santamaría, R. Rosario-Cruz. 2006․ Prevalence and potential risk factors for organophosphate and pyrethroid resistance in Boophilus microplus ticks on cattle ranches from the state of Yucatan, Mexico. Vet Parasitol 136, 335-442.

Rodríguez-Vivas RI, F Rodríguez-Arévalo, MA Alonso-Díaz, H FragosoSánchez, VM Santamaría, R Rosario-Cruz. 2006 . Prevalence and potential risk factors for amitraz resistance in Boophilus microplus ticks in cattle farms in the state of Yucatan, Mexico. Prev Vet Med $75,280-286$.

Sibson GJ. 1994. The effects of moxidectin against natural infestation of cattle (Boophilus microplus). Aust Vet J 71, 22-23.

Soberanes CN. 2007. El papel de la industria farmacéutica veterinaria en los programas de control de garrapatas y enfermedades transmitidas por garrapatas. Simposium Internacional: garrapatas, babesiosis y anaplasmosis. Tamaulipas, México, Pp 151-156.

Soutello RGV, MCZ Seno, AFT Amarante. 2007. Anthelmintic resistance in cattle nematodes in northwestern São Paulo State, Brazil. Vet Parasitol 148, 360-364.
Suárez VH, AL Lifschitz, JM Sallovitz, CE Lanusse. 2009. Effects of faecal residues of moxidectin and doramectin on the activity of arthropods in cattle dung. Ecotoxicol Environ Safety 72, 1551-1558.

Sumano LH, CL Ocampo. 2006. Farmacología Veterinaria. $3^{\text {ra }}$ ed. MacGrawHill Interamericana, México D.F., México, Pp 481-482.

Tedonkeng PE, F Tedonkeng, JR Kana, PV Khan, JB Boukila. 2005. A study of the acaricidal properties of an essential oil extracted from the leaves of Ageratum houstonianum. Cameroon. Vet Parasitol 128, 319-323.

Wardhaugh KG, P Holter, WA Whitby, K Shelley. 1996. Effect of drugs residues in the faeces of cattle treated with injectable formulations of ivermectin and moxidectin on larvae of the fly, Musca vettustissima and the house fly Musca domestica. Aust Vet $J$ 74, 370-374.

Wardhaugh KG, RJ Mahon. 1998. Comparative effects of abamectin and two formulations of ivermectin on the survival of larvae of dung breeding fly. Aust Vet $J$ 76, 270-272.

Willadsen P. 2001. The molecular revolution in the development of vaccines against ectoparasites. Vet Parasitol 101, 353-367. 